\title{
Limits and possibilities in a primary selective IgA deficiency treatment - case presentation
}

\author{
Monica Alexoae ${ }^{1}$, Mihaela Bataneant ${ }^{2}$, Ileana Ioniuc ${ }^{1}$, Alina Murgu' ${ }^{1}$ Alice Azoicai', \\ Aurica Rugina ${ }^{1}$ \\ ${ }^{1}$ Il Pediatric Clinic, "Gr. T. Popa" University of Medicine and Pharmacy, lasi, Romania \\ 2III Pediatric Clinic, "Victor Babes" University of Medicine and Pharmacy, Timisoara, Romania
}

\begin{abstract}
The primary deficit of IgA is the most common congenital immunodeficiency defined by the values of serum IgA below $0.07 \mathrm{~g} / \mathrm{l}$ and normal values of $\mathrm{IgG}$ and $\mathrm{IgM}$. Most cases are asymptomatic, only $10-15 \%$ is manifested by recurrent or persistent sinopulmonary infections, autoimmune diseases, allergies, malignancies. Some cases develop over the time a common variable immunodeficiency. The frequency of anti-IgA antibody justifies the cautiously use of transfusions of blood, plasma or intravenous immunoglobulin. The authors present a case of primary selective IgA deficiency, where the severity of infectious exacerbations appears to be due to the combination of a lack of IgG4, and discuss the possibilities and limits of therapy in light of recent data from the literature.
\end{abstract}

Keywords: primary selective IgA deficiency, IgG4 subclass

Primary immunodeficiencies are rare genetic diseases whose prevalence is not known. According to the European Society of immunodeficiency (ESID), the most frequent are the antibody immunodeficiency $-56.1 \%$ (common variable immunodeficiency, primary selective Ig A deficiency and the IgG subclass deficiency), followed by phagocytes defects $(11.2 \%), \mathrm{T}$ and $\mathrm{B}$ immune cell deficiencies $(8.4 \%)$, genetic diseases of immune regulation $(2.7 \%)$, deficits of the complement system $(2.5 \%)$, auto inflammatory diseases $(0.4 \%)$, defects on the receptors or signalling components $(0.4 \%)$. (1)

Primary selective IgA deficiency is the most common primary immunodeficiency in the Caucasian population. The incidence, underestimate because there are no screening programs, is different in relation to ethnicity (1:143 persons in Arabian Peninsula, 1:875 în UK, 1:14,800-18,500 in Japan) (2) The vast majority of cases (85-90\%) are asymptomatic, $10-15 \%$ is manifested by recurrent sinopulmonary infections, gastrointestinal disturbances, allergic diseases, autoimmune diseases and malignancies.

\section{CASE PRESENTATION}

JI, male, 8 years of age, from rural area, admitted for the first time in II Pediatric Clinic at the age of 6 and after with numerous presenting for periodic revaluation and exacerbation.

Familial history was insignificant for this case. Personal history: the second child in the family, from natural birth at 38 weeks, with 3,300 grams and $51 \mathrm{~cm}$; he received all national immunisation and also antipneumococal and rotaviral vaccination. He was in good health till the age of 2, and after he presented recurrent episodes of purulent pharyngitis, with multiple antibiotic cures or 7 to 10 days, with relapse after 3 days after the end of treatment. He underwent an adenotonsillectomy at the age of 3 and he also present rhinosinusitis and recurrent pneumopathies.

At the age of 6 years is hospitalised in II Pediatric Clinic, at the "Sf Maria" Emergency Hospital for Children, Iasi, with influenced general condition, normally developed for growth and height, mucocutaneous pallor, dental malposition and dystrophies, chest asymmetry (Fig. 1), pectus excava- 
tum, axillary limphadenophaty, right subscapular pigmented nevus, and 3 ferm, mobile, right subscapular tumoral formations with no adherence on the adjacent plans, ligamentar hyperlaxity (Fig. 2), cutis elastica (Fig. 3), nasal obstruction, muco purulent rhinorrhea, predominantly morning productive cough, crepitation and sibilants rales on both pulmonary areas.

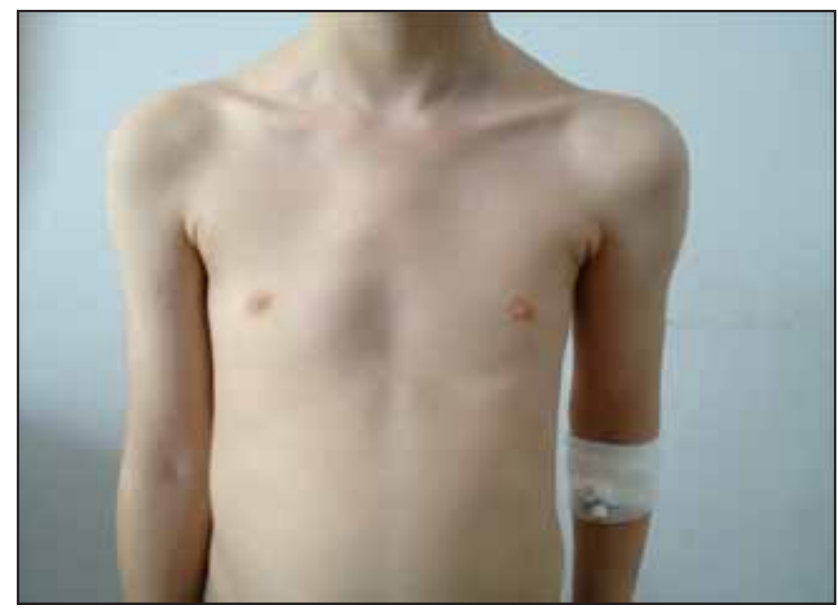

FIGURE 1. Chest asymmetry

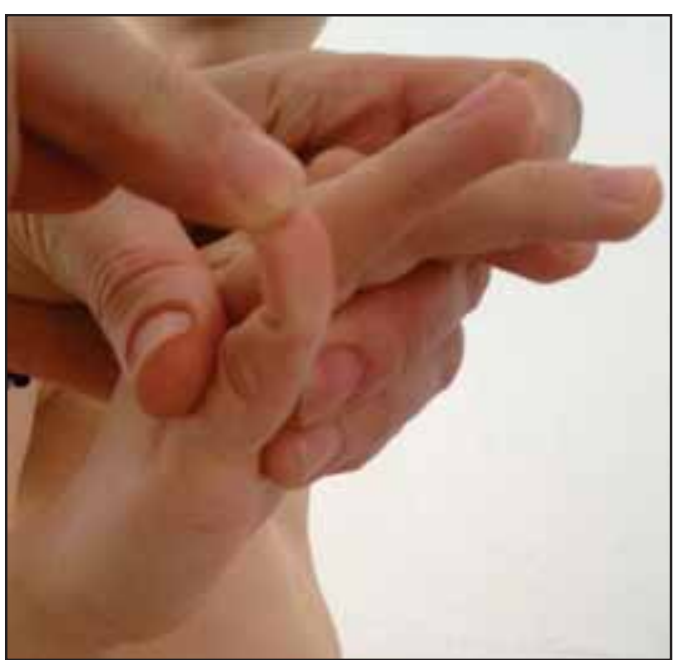

FIGURE 2. Ligamentary hyperlaxity

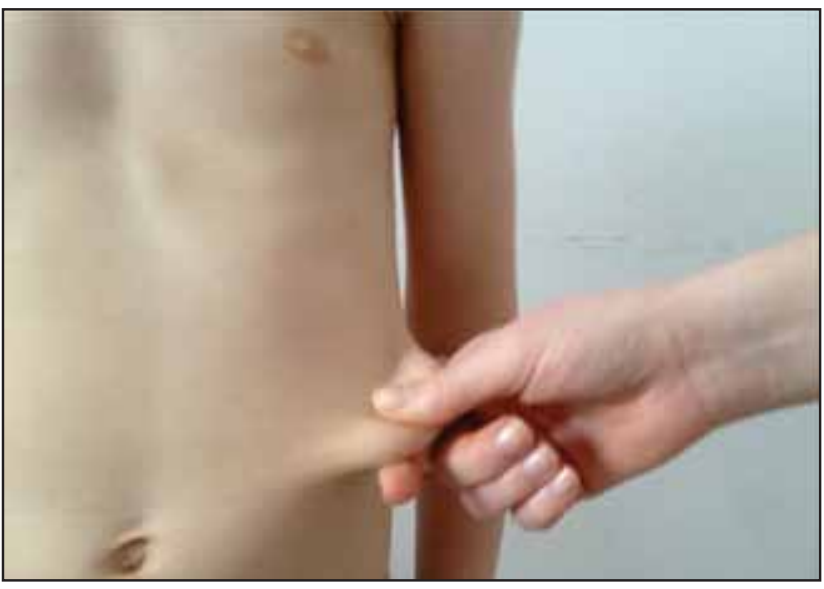

FIGURE 3. Cutis elastica
The recurrent infection diagnosis involved a complex investigation protocol: biologic, immunologic and imagistic in "Sf Maria" Emergency Hospital for Children, Iasi and "Louis Turcanu" Hospital for Children, Timisoara. Complete blood count and acute phase reactants had normal values. Serum protein electrophoresis was normal. Serum values of immunograme showed very low values of IgA level (under 0,05 g/l), normal values of $\mathrm{IgG}$ and IgM and low level of $\mathrm{IgG}_{4}$ subclass. The analysis of lymphocyte subpopulation documented decreased number of $\mathrm{CD}_{8} \mathrm{~T}$ lymphocyte and increased in $\mathrm{CD}_{4} / \mathrm{CD}_{8}$ ratio (Table 1 ). The dosage of the tetanus antibody titre showed a protective titre.

\section{TABLE 1. Immunological investigation}

\begin{tabular}{|l|c|}
\hline Immunograme & \\
Ig A (VN: 0.53-2.04) g/I & $<0,05$ \\
Ig G (6.98-15.6) & 14,88 \\
Ig M (0.31-1.79) & 1,56 \\
\hline Subclass IgG 1 (3,5-9,1 g/I) & 11 \\
gG 2 (0,85-3,3 g/l) & 1,3 \\
IgG 3 (0,20-1,04 g/I) & 0,83 \\
IgG 4 (0,030-1,577 g/I) & 0,02 \\
\hline Lymphocyte subclass & \\
Lymphocyte (2,000-2,700/mmc) & 2194 \\
Lymphocyte B (300-500/mmc) & 386 \\
Lymphocyte T (1,400-2,000/mmc) & 1152 \\
CD4+ (700-1,100/mmc) & 660 \\
CD8+ (600-900/mmc) & 335 \\
CD4/CD8 (1,1-1,4) & 1,97 \\
\hline
\end{tabular}

Chest CT scan examination confirmed the fault of the rib cage, with chest asymmetry and revealed a left lateral basal condensation, unsystematised bronchiectasis, one with air some other with mucoid impactation (Fig. 4, Fig. 5) and subcutaneous, homogeneous, iodophil, soft tissue formations in the rear wall of the right hemithorax.

Given the complexity of the clinical picture the investigation protocol was extended:

- associated bronchiectasis revealed by the CT scan imposed performimg a iontophoresis with normal values of $\mathrm{NaCl}(12 \mathrm{mmmol} / \mathrm{l})$.

- superficial and deep lymphadenopathies imposed the Toxoplasma, Brucella, Cytomegalovirus, HIV ${ }_{1,2}$ Bartonella Henselae serological exclusion (negative $\mathrm{IgG}$ and $\operatorname{IgM}$ antibody); Acute infection with Epstein Barr virus was confirmed by the positivity of IgM anti-VCA and anti-EBNA antibody.

- nasal swab, pharynx, sputum, faeces cultures, H. pylori antigen and fecal calprotectin were performed because of the infection and inflammation risk associated with IgA and IgG4 deficiency. The results were negatives. 

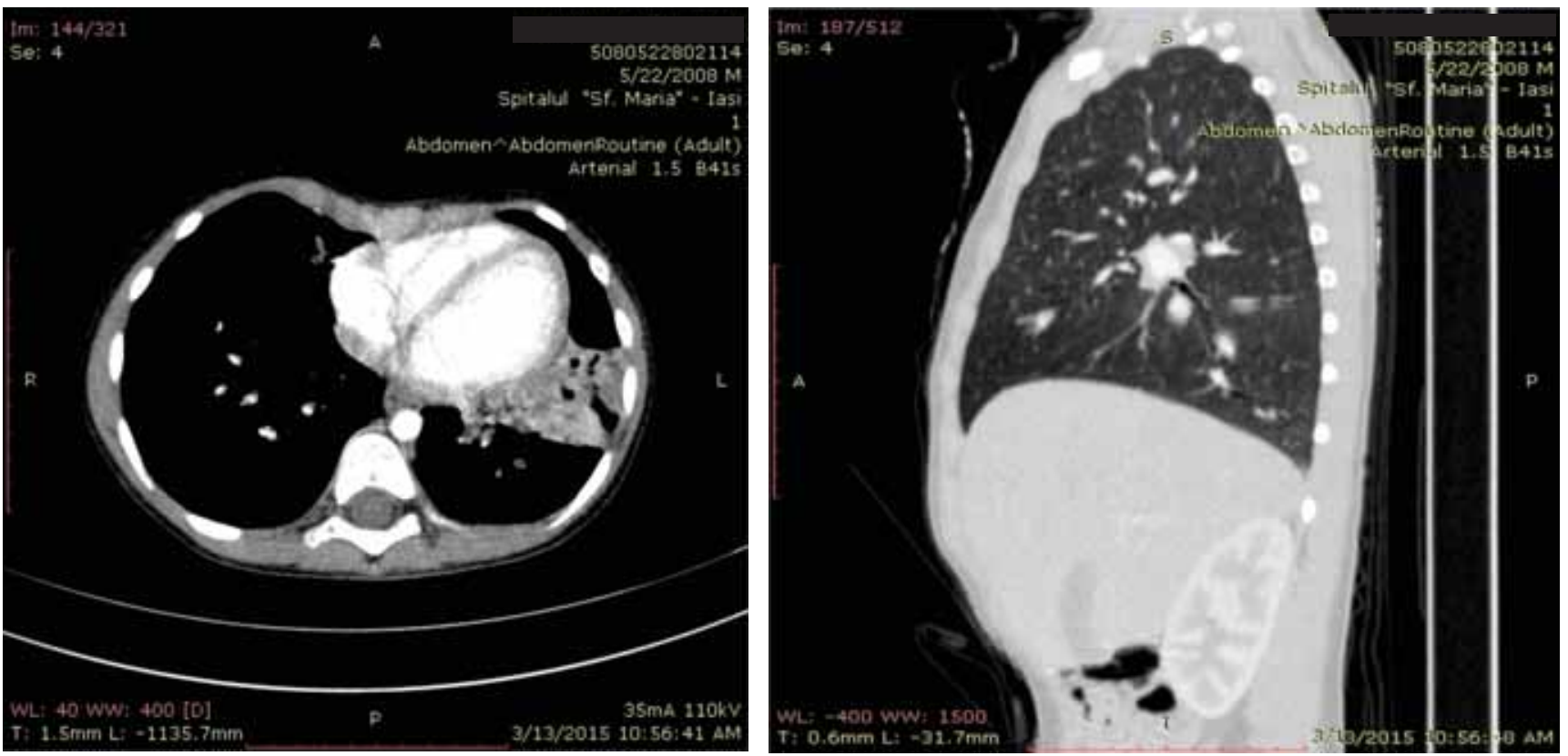

FIGURE 4, FIGURE 5. Unsystematised bronchiectasis

From carrying out the investigation, the final diagnosis in this case was:

- Primary selective IgA and IgG4 deficiency,

- Basal left pneumonia,

- Bilateral basal unsystematised bronchiectasis,

- Ehlers-Danlos syndrome - in observation (supported also by the geneticist),

- acute infection with Epstein-Barr virus.

Antibiotic, antiviral and symptomatic treatment was followed by surgical excision of one right interscapulum soft tissue tumoral formation, the histopathological appearance suggested an unspecific reactive follicular hyperplasia.

The clinical course was favorable. At the discharge, the patient received a special program for clinical control, and the parents were informed and counseled given the risk of the humoral immunodeficiency.

\section{DISCUSSIONS}

Selective IgA deficiency, the most common primary humoral immunodeficiency characterized by undetectable levels (less than $7 \mathrm{mg} / \mathrm{dl}$ ) serum concentrations of IgA in the presence of normal $\mathrm{IgG}$ and IgM titre. Diagnosis stated with certainty in children older than 4 years after exclusion of other causes of hypogammaglobulinemia, most often transient: infectious (HIV, infectious mononucleosis, congenital cytomegalovirus infection, Toxoplasma gondii and rubella), drugs, malignancy, systemic disease associated with excessive loss of immunoglobulins (nephrotic syndrome, extensive burns, bowel lymphangiectasis). (3)

The disease is asymptomatic in $85-90 \%$ of cases being diagnosed fortuitously, most commonly among people donating blood and only $10-15 \%$ is manifested by recurrent sinopulmonary infections. Susceptibility to infection is not correlated, in the most literature studies, with the severity of the IgA deficiency, but with concomitant deficiency of IgG4 and/or IgG2 and with an inadequate response to Pneumococcal polysaccharide vaccine and anti- Haemophilus type B vaccine (4). Other immunological conditions associated with primary IgA deficiency is the common variable immunodeficiency, the hyper-IgM syndrome, ataxia-telangiectasia syndrome, $18 \mathrm{q}$ deletion or $22 \mathrm{q} 11.2$, mucocutaneous candidiasis, complement and $\operatorname{IgE}$ deficiency.

In the presented case, capturing in the case evolution a CD8 lymphocytopenia impose careful follow-up for a possible associated complex combined immunodeficiency.

The primary IgA deficient patients have an increased risk of autoimmune diseases, particularly systemic lupus erythematosus (1-5\% of cases), rheumatoid arthritis (2-4\% of cases), celiac disease, diabetes mellitus, myasthenia gravis and autoimmune thyroiditis. Even in the absence of clinical symptoms, the prevalence of autoantibodies (rheumatoid factor, anticardiolipin antibodies, anti-nuclear antibodies) is greater than in the general population (5). 
There is no specific therapy for this primary immunodeficiency. Prophylactic antibiotics in children with recurrent infections is accepted, continuous or especially in cold season with the risk of secondary fungal and bacterial resistance development.

Classically, the primary IgA deficiency was considered as the main contraindication to the administration of intravenous immunoglobulin with the emergence of anti-IgA antibodies, the risk of severe systemic reactions being higher in terms of the association of IgG2 deficiency (3). Anti-IgA autoantibodies, most commonly IgG1 type, are present in a proportion of $9-44 \%$ in patients with primary IgA deficiency and the severe reactions can be triggered by blood transfusion, plasma and intravenous immunoglobulins. The risk of true anaphylactic reactions depends on the presence and the concentration of IgE type anti-IgA antibody (with lower prevalence) (6), their specificity, the amount of IgA in the product administered products and the route of administration (7).
Recent studies provide the safety of intravenous or subcutaneous administration of the product with low amounts of IgA (less than $0.5 \mathrm{mg} / \mathrm{l}$ ) in children with $\operatorname{IgA}$ deficiency and simultaneously $\mathrm{IgG}_{4}$ deficiency. The incidence of severe systemic side effects (dyspnea, fever, intense abdominal pain, myalgia and hypotension) recorded on a group of five children prospectively followed 170 received intravenous immunoglobulin was $5.3 \%$ without registering anaphylactic reactions (8). The effectiveness of these preparations analysed in terms of decrease in the frequency of infectious exacerbations is controversial, so that most authors recommended monthly therapy for 6 months, and then evaluation and interruption were is no improvement.

In conclusion, in children with IgA deficiency, the therapy requires prompt treatment of symptomatic infections possibly prophylactic antibiotics.

The presented case is particular by the association of clinical features: cutis laxa, ligament hyperlaxity and chest deformities, the $\mathrm{IgG}_{4}$ low values and also by the CD8 lymphocytopenia that impose careful follow-up.

Conflict of interest: none declared Financial support: none declared

6. Palmer D.S., O'Toole J., Montreuil T., Scalia V. et al. Screening of Canadian Blood Services donors for severe immunoglobulin $\mathrm{A}$ deficiency. Transfusion 2010; 50:1524-1531.

7. Rachid R., Bonilla F. The role of anti-lgA antibodies in causing adverse reactions to gamma globulin infusion in immunodeficient patients: A comprehensive review of the literature. Journal of Allergy and Clinical Immunol 2012; 130(2):553-554

8. Cunningham-Rundles C., Zhou Z., Mankarious S., Courier S. Longterm use of IgA-depleted intravenous immunoglobulin in immunodeficient subjects with anti-IgA antibodies. J Clin Immunol 1993; 13: $272-8$ 